https://helda.helsinki.fi

\title{
Local recovery of the compressional and shear speeds from the hyperbolic DN map
}

\section{Stefanov, Plamen}

2018-01

Stefanov , P , Uhlmann , G \& Vasy , A 2018 , ' Local recovery of the compressional and shear speeds from the hyperbolic DN map ' , Inverse Problems , vol. 34 , no. 1 , 014003 . https://doi.org/10.1088/136

http://hdl.handle.net/10138/237258

https://doi.org/10.1088/1361-6420/aa9833

cc_by

Downloaded from Helda, University of Helsinki institutional repository.

This is an electronic reprint of the original article.

This reprint may differ from the original in pagination and typographic detail.

Please cite the original version. 
PAPER

Local recovery of the compressional and shear speeds from the hyperbolic DN map

To cite this article: Plamen Stefanov et al 2018 Inverse Problems 34014003

View the article online for updates and enhancements.

\section{Related content}

\author{
Thermoacoustic tomography arising in \\ brain imaging \\ Plamen Stefanov and Gunther Uhlmann \\ Multiwave tomography in a closed domain: \\ averaged sharp time reversal \\ Plamen Stefanov and Yang Yang \\ - Thermoacoustic tomography \\ Plamen Stefanov and Gunther Uhlmann
}

\section{Recent citations}

- The first 100 years of the Radon transform Ronny Ramlau and Otmar Scherzer

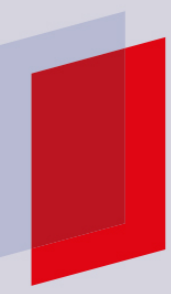

\section{IOP ebooks}

Bringing you innovative digital publishing with leading voices to create your essential collection of books in STEM research. Start exploring the collection - download the first chapter of every title for free. 


\title{
Local recovery of the compressional and shear speeds from the hyperbolic DN map
}

\author{
Plamen Stefanov ${ }^{1,6,7}$,, Gunther UhImann ${ }^{2,3,4,6}$ \\ and Andras Vasy ${ }^{5,6}$ \\ 1 Department of Mathematics, Purdue University, West Lafayette, IN 47907, \\ United States of America \\ ${ }^{2}$ Department of Mathematics, University of Washington, Seattle, WA 98195, \\ United States of America \\ ${ }^{3}$ Department of Mathematics, University of Helsinki, Finland \\ ${ }^{4}$ IAS, HKUST, Clear Water Bay, Hong Kong, People's Republic of China \\ 5 Department of Mathematics, Stanford University, Stanford, CA 94305, \\ United States of America \\ E-mail: stefanov@math.purdue.edu
}

Received 13 June 2017, revised 30 October 2017

Accepted for publication 3 November 2017

Published 12 December 2017

\section{Abstract}

We study the isotropic elastic wave equation in a bounded domain with boundary. We show that local knowledge of the Dirichlet-to-Neumann map determines uniquely the speed of the p-wave locally if there is a strictly convex foliation with respect to it, and similarly for the s-wave speed.

Keywords: compressional and shear speeds, hyperbolic DN map, isotropic elastic wave equation

(Some figures may appear in colour only in the online journal)

\section{Introduction}

Consider the isotropic elastic wave equation in a smooth bounded domain $\Omega$. We study the following problem: can we determine the Lamé parameters $\lambda, \mu$ and the density $\rho$ from the knowledge of the Dirichlet-to-Neumann $\Lambda$ (DN) map on the boundary? In fact, we are interested in the local problem: local recovery of those parameters from local or even microlocal information about $\Lambda$. Our main motivation is the local seismology problem of recovery of the inner structure of the Earth from local measurements on its surface of seismic waves.

\footnotetext{
${ }^{6}$ The authors were partially supported by the National Science Foundation under grant DMS-1600327 (P.S.), DMS-1265958 (G.U.) and DMS-1361432 (A.V.).

${ }^{7}$ Author to whom any correspondence should be addressed. 
This problem is well studied for the wave equation $\left(\partial_{t}^{2}-\Delta_{g}\right) u=0$ related to a Riemannian metric $g$ with either full or partial boundary data. It is known that one can recover $g$ up to an isometry fixing $\partial \Omega$ pointwise [5], using the boundary control method developed by [2]. The latter relies on the unique continuation result of Tataru [26]. We refer to [13] for related results and more references. Logarithmic type of stability is proved in [7, 14]. Hölder type of stability estimates with full data have been proven in [1, 6, 17, 22] and most recently in [25], under some assumptions on the metric, for example absence of conjugate points.

In the elastic case, the results are less complete. Unique continuation holds [10] but the boundary control method is not known to work, see, e.g. [4]. The reason is that it is not possible, or at least not known how to decouple the elasticity system completely even though it is easy to do that on the principal symbol level or even for the full symbol, see (21) below, but only microlocally. A Lamé type of system having the same principal part which can be decoupled fully was studied in [3] and the boundary control method was used for it for a unique recovery of the two wave speeds locally with a local data. Numerical reconstruction is proposed in [15].

Rachelle [18] proved that one can recover the jet of $\lambda, \mu$ and $\rho$ at $\partial \Omega$ explicitly. In [19, 20], she showed that one can recover those three parameters in $\Omega$ provided that $\Lambda$ is known on the whole boundary and assuming that the two wave speeds (2) are simple (strict convexity and no conjugate points). The proof is based on recovering the lens relations related to the two speeds and then applying known rigidity results. The recovery of all the three parameters in [20] requires the study of the second order term and an inversion of the geodesic ray transform. The second author and Hansen [11] studied this problem with a residual stress and without the assumption of no-conjugate points or caustics and showed that one can recover both lens relations and derive several consequences of that.

In this work we show that one can recover uniquely the two wave speeds $c_{p}$ and $c_{s}$, see (2), locally under the assumption of existence of a strictly convex foliation. This condition allows for conjugate points, see, e.g. [16]. If $\Omega$ is a ball and the speeds increase when the distance to the center decreases (typical for geophysical applications), the foliation condition is satisfied, see section 6. To prove the main result, we show that one can recover the lens relations related to the two speeds in an explicit way and then apply the results of the authors [23], see also [24], about local recovery of a sound speed in the acoustic equation from the associated lens relation, also known locally. Those works are based in particular on a method developed in [29] to prove a uniqueness and a stability result for the linear problem of recovery of a function from its geodesic ray transform known locally.

That argument also implies stability as a consequence of the stability result in [23] but we do not make this formal. It also implies a theoretical reconstruction following the procedure in [29]. Also, we can apply the result if there is an internal closed strictly convex surface where the coefficients jump, like in the elastic Earth model, and recover the two speeds between the boundary and that surface. Indeed, for that we only need the lens relation along geodesics not hitting that surface; and that can be extracted from the microlocal support of the DN map. Note that we recover two quantities depending on the three parameters $\lambda, \mu$ and $\rho$. Recovery of all three parameters would require analysis of the amplitude in the geometric optics construction, similarly to what is done in [20].

\section{Main result}

The isotropic elastic system in a smooth bounded domain $\Omega \subset \mathbf{R}^{3}$ is described as follows. The elasticity tensor is defined by

$$
c_{i j k l}=\lambda \delta_{i j} \delta_{k l}+\mu\left(\delta_{i k} \delta_{j l}+\delta_{i l} \delta_{j k}\right),
$$


where $\lambda>0, \mu>0$ are the Lamé parameters belonging to $C^{\infty}(\bar{\Omega})$. The elastic wave operator is given by

$$
(E u)_{i}=\rho^{-1} \sum_{j k l} \partial_{j} c_{i j k l} \partial_{l} u_{k},
$$

where $0<\rho \in C^{\infty}(\bar{\Omega})$ is the density and the vector function $u$ is the displacement.

The operator $E$ is symmetric on $L^{2}\left(\Omega ; \mathbf{C}^{3}, \rho \mathrm{d} x\right)$. It has has a principal symbol

$$
\sigma_{p}(-E) v=\frac{\lambda+\mu}{\rho} \xi \xi \cdot v+\frac{\mu}{\rho}|\xi|^{2} v, \quad v \in \mathbf{C}^{3} .
$$

Taking $v=\xi$ and $v \perp \xi$, we recover the well known fact that that $\sigma_{p}(-E)$ has eigenvalues

$$
c_{p}=\sqrt{(\lambda+2 \mu) / \rho}, \quad c_{s}=\sqrt{\mu / \rho}
$$

of multiplicities 1 and 2, respectively and eigenspaces $\mathbf{R} \xi$, and $\xi^{\perp}$. Those are known as the speeds of the p-waves and the s-waves, respectively. The eigenspaces correspond to the polarization of those waves. The characteristic variety $\operatorname{det} \sigma_{p}(E)=0$ is the union of $\Sigma_{p}:=\left\{\tau^{2}=c_{p}^{2}|\xi|^{2}\right\}$ and $\Sigma_{s}:=\left\{\tau^{2}=c_{s}^{2}|\xi|^{2}\right\}$, each one having two connected components (away from the zero section), determined by the sign of $\tau$.

Let $u$ solve the elastic wave equation

$$
\left\{\begin{array}{c}
u_{t t}-E u=0 \quad \text { in } \mathbf{R} \times \Omega, \\
\left.u\right|_{\mathbf{R} \times \partial \Omega}=f, \\
\left.u\right|_{t<0}=0,
\end{array}\right.
$$

with $f$ given so that $f=0$ for $t<0$. The DN $\Lambda$ map is defined by

$$
(\Lambda f)_{i}=(N u)_{i}:=\left.\Sigma_{j} \sigma_{i j}(u) \nu^{j}\right|_{\partial \Omega}
$$

where $\nu$ is the outer unit normal on $\partial \Omega$, and $\sigma_{i j}(u)$ is the stress tensor

$$
\sigma_{i j}(u)=\lambda \nabla \cdot u \delta_{i j}+\mu\left(\partial_{j} u_{i}+\partial_{i} u_{j}\right) .
$$

Note that $E u=\rho^{-1} \delta \sigma(u)$, where $\delta$ is the divergence of the 2-tensor $\sigma(u)$.

We introduce the notion of a convex foliation with respect to some metric $g$ now. Let $\Omega_{\mathrm{ext}}$ be an open domain containing $\bar{\Omega}$ and extend the coefficients there in a smooth way; and let $g$ be a Riemannian metric there.

Definition 1. Let $\kappa: \Omega_{\mathrm{ext}} \rightarrow \mathbf{R}$ be a smooth function which level sets $\kappa^{-1}(q), q \leqslant 1$, restricted to $\bar{\Omega}$, are strictly convex viewed from $\kappa^{-1}((-\infty, q))$ w.r.t. $g ; d \kappa \neq 0$ on these level sets, and $\kappa^{-1}(0) \cap \bar{\Omega} \subset \partial \Omega$. We call $\kappa^{-1}(q) \cap \bar{\Omega}, q \in[0,1]$, a strictly convex foliation of $\kappa^{-1}([0,1]) \cap \bar{\Omega}$ with respect to $g$.

An example is shown in figure 1, where, for illustration purposes, level sets which are Euclidean strictly convex should be assumed to be strictly convex w.r.t. $g$ as well. One special case is to take $\kappa^{-1}(0)=\partial \Omega$ if $\partial \Omega$ is strictly convex w.r.t. $g$. We recall that an oriented hypersurface $S$ is strictly convex w.r.t. $g$ (when viewed from the side determined by the normals consistent with the orientation) if the second fundamental form on $S$ is positive. An equivalent definition is to require every geodesic $\gamma(t)$ tangent to $S$ at $\gamma(0) \in S$ to stay on that side (from which we view $S$ ) for $|t| \ll 1$ with the distance to $S$ satisfying $\operatorname{dist}(\gamma(t), S) \geqslant t^{2} / C$ for $|t| \ll 1$.

We introduce the lens rigidity problem next. For a compact manifold $(M, g)$ with a boundary, let the manifolds $\partial_{ \pm} S M$ consist of all vectors $(x, v)$ with $x \in \partial M, v$ unit in the metric $g$, and pointing outside/inside $M$. We define the scattering relation 


$$
L: \partial_{-} S M \longrightarrow \partial_{+} S M
$$

in the following way: for each $(x, v) \in \partial_{-} S M, L(x, v)=(y, w)$, where $(y, w)$ are the exit point and direction, if exist, of the maximal unit speed geodesic $\gamma_{x, v}$ in the metric $g$, issued from $(x, v)$. Let

$$
\ell: \partial_{-} S M \longrightarrow \mathbf{R} \cup \infty
$$

be its length, possibly infinite. If $\ell<\infty$, we call $M$ non-trapping. The maps $(L, \ell)$ together are called lens relation (or lens data).

It is convenient to identify vectors in $\partial_{-} S M$ with their projections on the unit ball bundle $B(\partial M)$; and similarly for $\partial_{+} S M$. Then we can view $L$ and $\ell$ as maps from $B(\partial M)$ to itself; or from $B(\partial M)$ to $\mathbf{R} \cap \infty$, respectively.

Below, we denote by $L_{p}$ and $L_{s}$ the lens relations in $M=\bar{\Omega}$ w.r.t. the metrics $c_{p}^{-2} \mathrm{~d} x^{2}$ and $c_{s}^{-2} \mathrm{~d} x^{2}$, respectively. Similarly, we denote the corresponding $\ell^{\prime}$ s by $\ell_{p}$ and $\ell_{s}$.

Theorem 1. Let $\rho, \lambda$, $\mu$ be smooth in $\bar{\Omega}$. Let $\kappa^{-1}(q), q \in[0,1]$ be a strictly convex foliation w.r.t. $c_{p}$, and let $\Gamma=\kappa^{-1}([0,1]) \cap \partial \Omega$. Then for every $\varepsilon>0, c_{p}$ is uniquely determined on the foliation $\kappa^{-1}([0,1]) \cap \bar{\Omega}$ by knowledge, up to a smooth function, of the kernel $\Lambda\left(t_{2}, x_{2}, t_{1}, x_{1}\right)$ of $\Lambda$ on $(0, T) \times \Gamma \times(0, \varepsilon) \times \Gamma$, if $T$ is greater than the length of all geodesics, in the metric $c_{p}^{-2} \mathrm{~d} x^{2}$, in $\bar{\Omega}$ having the property that each one is tangent to some of the hypersurfaces in the foliation.

The same statement remains true for $c_{p}$ replaced by $c_{s}$.

We refer to figure 1 for an illustration of the set where we can recover the speeds. Note that we do not require to know the actual (localized) DN map; we just need to know where the singularities of the kernel are in space and time, see also figure 2.

We only need microlocal information about $\Lambda$, wee remark 5.1. Also, since $\left(L_{p}, \ell_{p}\right)$ and $\left(L_{s}, \ell_{s}\right)$ are part of the wave front set of the kernel of $\Lambda$, we can argue that they can be recovered stably form it; and in fact, they are directly observable in seismic experiments. Then one can apply the stability result in [23] to conclude that $c_{s}$ and $c_{p}$ are stably recoverable from $\Lambda$. We will not make this statement more precise in this paper.

\section{Preliminaries}

\subsection{An invariant formulation}

We have

$$
(E u)_{i}=\rho^{-1} \sum_{j}\left(\partial_{i} \lambda \partial_{j} u_{j}+\partial_{j} \mu \partial_{j} u_{i}+\partial_{j} \mu \partial_{i} u_{j}\right)
$$

This can also be written in the following divergence form.

$$
E u=\rho^{-1}\left(\mathrm{~d} \lambda \delta u+2 \delta \mu \mathrm{d}^{s} u\right),
$$

where $\mathrm{d}^{s} u=\left(\partial_{j} u_{i}+\partial_{i} u_{j}\right) / 2$ is the symmetric differential, and $\delta=-\left(\mathrm{d}^{s}\right)^{*}$ is the divergence of symmetric fields.

To prepare ourselves for changes of variables needed in the analysis near surfaces that we will flatten out, we will write $E$ in invariant way in the presence of a Riemannian metric $g$. We view $u$ as an one form (a covector field) and we define the symmetric differential $\mathrm{d}^{s}$ and the divergence $\delta$ by 


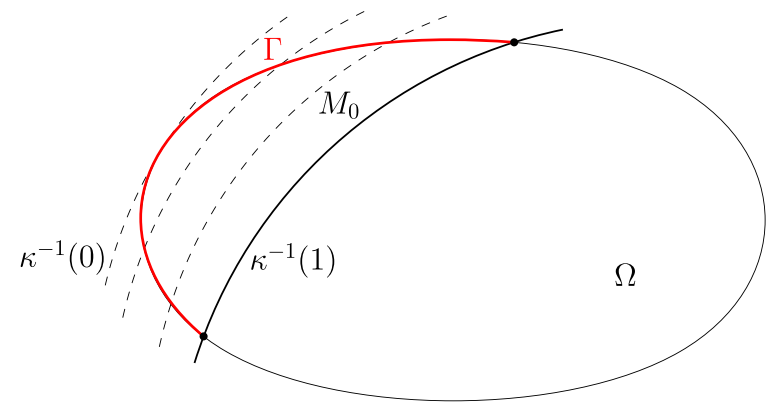

Figure 1. The foliation related to either $c_{p}$ and $c_{s}$, and the part $\Gamma$ of $\partial \Omega$ where $\Lambda$ is known. We can recover the speed in $M_{0}$.

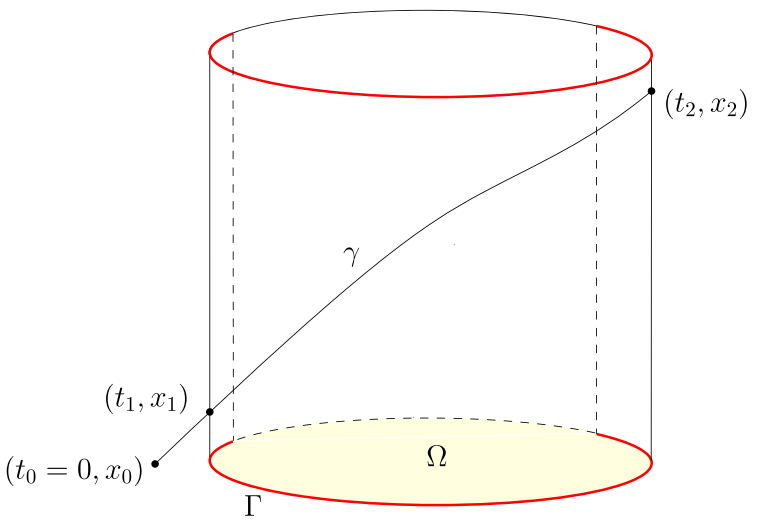

Figure 2. The bicharacteristic $\gamma$ (projected to the base).

$$
\left(\mathrm{d}^{s} u\right)_{i j}=\frac{1}{2}\left(\nabla_{i} u_{j}+\nabla_{j} u_{i}\right), \quad(\delta v)_{i}=\nabla^{j} v_{i j}, \quad \delta u=\nabla^{i} u_{i},
$$

where $\nabla$ is the covariant differential, $\nabla^{j}=g^{i j} \nabla_{i}, u$ is a covector field, and $v$ is a symmetric covariant tensor field of order two. Note that $\mathrm{d}^{s}$ increases the order of the tensor by one while $\delta$ decreases it by one. Then we define $E$ by (8). The stress tensor (5) is given by

$$
\sigma(u)=\lambda(\delta u) g+2 \mu \mathrm{d}^{s} u,
$$

and then $E u=\rho^{-1} \delta \sigma(u)$. The Neumann boundary condition $N u$ at $\partial \Omega$ is still given by prescribing the values of $\sigma_{i j}(u) \nu^{j}$ on it as in (4). The operator $E$, defined originally on $C_{0}^{\infty}(\Omega)$ extends to a self-adjoint operator in $L^{2}(\Omega, \rho \mathrm{d} x)$. This extension is the one satisfying the zero Dirichlet boundary condition on $\mathbf{R} \times \partial \Omega$. In particular, this shows that the mixed problem (3) is solvable with smooth data $f$ at least since one can always extend $f$ inside and reduce the problem to solving one with a zero boundary condition and a non-zero source term; and then use the Duhamel's principle for the latter.

We show next that the data $(u, N u)$ on the boundary is equivalent to knowing the Cauchy data on it, see also [18, section 3.1]. In next lemma, we use semigeodesic coordinates $x=\left(x^{\prime}, x^{3}\right)$ to a given hypersurface $S$, with $x^{3}>0$ on one side of it, defining the orientation. The Euclidean metric then takes the form $g$ in those coordinates with $g_{\alpha 3}=\delta_{\alpha 3}$ for $1 \leqslant \alpha \leqslant 3$. 
Lemma 1. For every hypersurface $S$, the pair $\left.(u, N u)\right|_{S}$ determined uniquely the Cauchy data $\left.\left(u, \partial_{\nu} u\right)\right|_{s}$. More precisely, in semigeodesic coordinates, the normal derivative of $u$ on $S$ can be obtained from $\left.u\right|_{S}$ and $\left.\mathrm{Nu}\right|_{S}$ by the relations

$$
\begin{aligned}
\partial_{3} u_{\alpha} & =\frac{1}{\mu}(N u)_{\alpha}-\partial_{\alpha} u_{3}+2 \Gamma_{j 3}^{k} u_{\alpha}, \quad \alpha=1,2, \\
\partial_{3} u_{3} & =\frac{1}{2 \mu}\left((N u)_{3}-\lambda(\delta u)\right),
\end{aligned}
$$

where $\Gamma_{i j}^{k}$ are the Christoffel symbols of the Euclidean metric in semigeodesic coordinates.

Before presenting the proof, we want to emphasize that $u$ is also transformed in the new coordinates (as a covector), and $\delta$ is the divergence w.r.t. the transformed metric. Also, in more invariant terms, (10) takes the form

$$
\begin{aligned}
\nabla_{3} u_{\alpha} & =\frac{1}{\mu}(N u)_{\alpha}-\nabla_{\alpha} u_{3}, \quad \alpha=1,2, \\
\partial_{3} u_{3} & =\frac{1}{2 \mu}\left((N u)_{3}-\lambda(\delta u)\right),
\end{aligned}
$$

where $\nabla$ is the covariant derivative, and we used the fact that $\nabla_{3} u_{3}=\partial_{3} u_{3}$.

Proof. In those coordinates,

$$
(N u)_{j}=\lambda(\delta u) \delta_{j 3}+\mu\left(\partial_{3} u_{j}+\partial_{j} u_{3}-2 \Gamma_{j 3}^{k} u_{k}\right) .
$$

Setting $j=3$, we get the second formula in (10) because $\Gamma_{33}^{k}=0$. Taking $j=1,2$, we get the first one one.

\section{Geometric optics for the elastic wave equation}

We recall the well known geometric optics construction for the acoustic and the elastic wave equations, see, e.g. [27, 28].

\subsection{The Cauchy problem with data at $t=0$ in the acoustic case}

We start with the scalar acoustic case which we use in the analysis of the elastic one. We work in arbitrary dimensions $n \geqslant 2$ here. Details can be found in [27, 28], for example. In fact, for the elastic case, we need to study (12) for vector valued functions with a first order $\Psi$ DO term and this case is considered in [27]. While the leading order amplitude depends on the subprincipal term of the equation; the geometry of the problem, i.e. the propagation of singularities is the same as that for (12).

We recall briefly the geometric optic construction for the acoustic wave equation

$$
\left(\partial_{t}^{2}-c^{2} \Delta_{g_{0}}\right) u=0
$$

with Cauchy data $\left(u, \partial_{t} u\right)=\left(h_{1}, h_{2}\right)$ at $t=0$. Here, $g_{0}$ is a Riemannian metric that we include in order to have the flexibility to change coordinates easily; and $\Delta_{g_{0}}$ is the Laplace-Beltrami operator. Up to lower order terms, $c^{2} \Delta_{g_{0}}$ coincides with $\Delta_{g}$ with $g:=c^{-2} g_{0}$. We are looking for solutions of the form 


$$
\begin{array}{r}
u(t, x)=(2 \pi)^{-n} \sum_{\sigma= \pm} \int \mathrm{e}^{\mathrm{i} \phi_{\sigma}(t, x, \xi)}\left(a_{1, \sigma}(t, x, \xi) \hat{h}_{1}(\xi)\right. \\
\left.+|\xi|_{g_{0}}^{-1} a_{2, \sigma}(t, x, \xi) \hat{h}_{2}(\xi)\right) \mathrm{d} \xi
\end{array}
$$

modulo terms involving smoothing operators of $h_{1}$ and $h_{2}$, defined in some neighborhood of $t=0, x=x_{0}$ with some $x_{0}$. This parametrix differs from the actual solution by a smoothing operator applied to $\mathbf{h}=\left(h_{1}, h_{2}\right)$, as it follows from standard hyperbolic estimates.

Here, $a_{j, \sigma}$ are classical amplitudes of order zero depending smoothly on $t$ of the form

$$
a_{j, \sigma} \sim \sum_{k=0}^{\infty} a_{j, \sigma}^{(k)}, \quad \sigma= \pm, j=1,2,
$$

where $a_{j, \sigma}^{(k)}$ is homogeneous in $\xi$ of degree $-k$ for large $|\xi|$. The phase functions $\phi_{ \pm}$are positively homogeneous of order 1 in $\xi$ solving the eikonal equations

$$
\partial_{t} \phi \pm c(x)\left|\nabla_{x} \phi\right|_{g_{0}}=0,\left.\quad \phi_{ \pm}\right|_{t=0}=x \cdot \xi .
$$

Such solutions exist locally only, in general.

Equate the order 1 terms in the expansion of $\left(\partial_{t}^{2}-c^{2} \Delta_{g_{0}}\right) u$ to get that the principal terms of the amplitudes must solve the transport equation

$$
\left(\left(\partial_{t} \phi_{ \pm}\right) \partial_{t}-c^{2} g_{0}^{i j}\left(\partial_{i} \phi_{ \pm}\right) \partial_{j}+C_{ \pm}\right) a_{j, \pm}^{(0)}=0
$$

with appropriate initial conditions and

$$
2 C_{ \pm}=\left(\partial_{t}^{2}-c^{2} \Delta_{g_{0}}\right) \phi_{ \pm}
$$

Equating terms homogeneous in $\xi$ of lower order we get transport equations for $a_{j, \sigma}^{(k)}, j=1,2, \ldots$ with the same left-hand side as in (16) with a right-hand side determined by $a_{k, \sigma}^{(k-1)}$.

The transport equations are ODEs along the zero bicharacteristics, which are just the geodesics of the metric $g$ lifted to the phase space, with vectors identified by covectors by the metric. The integrals appearing in (13) are Fourier integral operators (FIOs) either with $t$ considered as a parameter, or as $t$ considered as one of the variables. In the former case, singularities of $\left(h_{1}, h_{2}\right)$ propagate along the zero bicharacteristics. More precisely, for every $t$,

$$
\mathrm{WF}(\mathbf{u}(t, \cdot))=C_{+}(t) \circ \mathrm{WF}(\mathbf{h}) \cup C_{-}(t) \circ \mathrm{WF}(\mathbf{h}),
$$

where $\mathbf{u}:=\left(u, u_{t}\right), \mathbf{h}=\left(h_{1}, h_{2}\right)$ and

$$
\begin{aligned}
& C_{+}(t)(x, \xi)=\left(\gamma_{x, \xi /|\xi|_{g}}(t),|\xi|_{g} g \dot{\gamma}_{x, \xi /|\xi|_{g}}(t)\right), \\
& C_{-}(t)(x, \xi)=\left(\gamma_{x,-\xi /|\xi|_{g}}(t),-|\xi|_{g} g \dot{\gamma}_{x,-\xi /|\xi|_{g}}(t)\right)=C_{+}(-t)(x, \xi),
\end{aligned}
$$

and for $(x, \eta) \in T^{*} \mathbf{R}^{3} \backslash 0, \gamma_{x, \eta}$ is the geodesic issued from $x$ in direction $g^{-1} \eta$.

On the other hand, considering $t$ as one of the variables,

$$
\mathrm{WF}(\mathbf{u})=C_{+} \circ \mathrm{WF}(\mathbf{h}) \cup C_{-} \circ \mathrm{WF}(\mathbf{h}),
$$

where

$$
\begin{aligned}
& C_{+}(x, \xi)=\left\{\left(t, \gamma_{x, \xi /|\xi|_{g}}(t),-|\xi|_{g},|\xi|_{g} g \dot{\gamma}_{x, \xi /|\xi|_{g}}(t)\right), t \in \mathbf{R}\right\}, \\
& C_{-}(x, \xi)=\left\{\left(t, \gamma_{x,-\xi /|\xi|_{g}}(t),|\xi|_{g},-|\xi|_{g} g \dot{\gamma}_{x,-\xi /|\xi|_{g}}(t)\right) t \in \mathbf{R}\right\} .
\end{aligned}
$$


In the analysis below, we will consider $C_{+}$only.

The construction above can be done in some neighborhood of a fixed point $\left(0, x_{0}\right)$ in general. To extend it globally, we can localize it first for $\mathbf{h}$ with $\mathrm{WF}(\mathbf{h})$ in a conic neighborhood of some fixed $\left(x_{0}, \xi^{0}\right) \in T^{*} \mathbf{R}^{3} \backslash 0$. Then $u$ will be well defined near the geodesic issued from that point but in some neighborhood of $\left(0, x_{0}\right)$ in general. We can fix some $t=t_{1}$ at which $u$ is still defined, take the Cauchy data there and use it to construct a new solution. Then we get an FIO which is a composition of the two local FIOs each one associated with a canonical diffeomorphism, then so is the composition. Then we can use a partition of unity to conclude that while the representation (13) is local, the conclusions (18) and (19) are global. In fact, it is well known that both $\mathbf{h} \mapsto \mathbf{u}$ and $\mathbf{h} \mapsto \mathbf{u}(t, \cdot)$ with $t$ fixed are global FIOs associated with the canonical relations in (18) and (19).

In particular, if $S$ is a smooth hypersurface, and $\gamma_{x, \xi}(t)$ hits $S$ for the first time $t=t(x, \xi)$ transversely locally, then $\left.\mathbf{h} \mapsto u\right|_{S}$ is an FIO again with a canonical relation as $C_{+}$above but with $t=t(x, \xi)$ and $\dot{\gamma}$ replaced by its tangential projection $\eta^{\prime}:=\dot{\gamma}^{\prime}$. Notice that $\tau=-|\xi|_{g}<0$ for $C_{+}$and $\tau=|\xi|_{g}>0$ for $C_{-}$. Also, $\left|\tau^{\prime}\right|<|\eta|_{g}$ with equality for tangent rays which we exclude.

\subsection{The Cauchy problem at $t=0$ and propagation of singularities in the elastic case}

Consider the elastic wave equation

$$
\begin{aligned}
u_{t t}-E u & =0, \\
\left.\left(u, u_{t}\right)\right|_{t=0} & =\left(h_{1}, h_{2}\right)
\end{aligned}
$$

with Cauchy data $\mathbf{h}:=\left(h_{1}, h_{2}\right)$ at $t=0$. We want to solve it microlocally for $t$ in some interval and $x$ in an open set.

We recall the construction in [27]. The principal symbol $\sigma_{p}(-E)$ of $-E$ has eigenvalues of constant multiplicities. Near every $\left(x_{0}, \xi^{0}\right) \in T^{*} \bar{\Omega} \backslash 0$, one can decouple the full symbol $\sigma(-E)$ fully up to symbols of order $-\infty$. In other words, there exist an elliptic matrix valued $\Psi$ DO $U$ of order 0 microlocally defined near $\left(x_{0}, \xi^{0}\right)$, so that

$$
U^{-1} E U=\left(\begin{array}{ll}
P & 0 \\
0 & S
\end{array}\right)
$$

modulo $S^{-\infty}$ near $\left(x_{0}, \xi^{0}\right)$, where $P$ is scalar, and $S$ is a $2 \times 2$ matrix symbol, with principal symbols $\sigma_{p}(P)=c_{p}^{2}|\xi|^{2}, \sigma_{p}(S)=c_{s}^{2}|\xi|^{2}$. In other words, $P$ is scalar and $S$ is principally scalar. The inverse $U^{-1}$ above stands for a microlocal parametrix. In fact, $U$ can be chosen to be unitary in microlocal sense [21]. As an example, the principal symbol of $U$ can be chosen to be

$$
\sigma_{p}(U)=|\xi|^{-1}\left(\begin{array}{ccc}
\xi_{1} & -\xi_{2} & 0 \\
\xi_{2} & \xi_{1} & \xi_{3} \\
\xi_{3} & 0 & -\xi_{2}
\end{array}\right)
$$

when $\xi_{2} \neq 0$. It then follows that microlocally, the elasticity system decouples into two wave equations of the kind (22), the first one scalar, and the second one a $2 \times 2$ system. Indeed, set $\left(w_{p}, w_{s}\right)=U^{-1} u$ with $w_{p}$ scalar and $w_{s}$ two-vector valued. Then, microlocally, in the conic set where (21) holds, (20) reduces to

$$
\left(\partial_{t}^{2}-c_{p}^{2} \Delta-R_{p}\right) w_{p} \in C^{\infty}, \quad\left(\partial_{t}^{2}-c_{s}^{2} \Delta-R_{s}\right) \in C^{\infty},
$$

where $R_{p}$ and $R_{s}$ are $\Psi$ DOs of order one, with Cauchy data at $t=0$ which can be obtained from that for $u$. The system (22) is coupled in the smoothing part only which is moved to the 
right hand side above. The first equation has $\Sigma_{p}$ as a characteristic manifold, while the second one has $\Sigma_{s}$. Now, we can apply the propagation of singularities results, and more specifically those in $[8,27]$ reviewed in section 4.1 . Then we conclude that singularities in those neighborhoods propagate along the zero bicharacteristics of $\tau^{2}-c_{p}^{2}|\xi|^{2}$ and $\tau^{2}-c_{s}^{2}|\xi|^{2}$, respectively. This implies a global result, as well. We get a bit more: (22) is microlocally equivalent to (20).

\section{Proof of the main results}

The following theorem is a local version of the statement that given $\Lambda$, we can recover the lens relations $\left(L_{p}, \ell_{p}\right)$ and $\left(L_{s}, \ell_{s}\right)$, see $[11,19]$ for the global version.

Theorem 2. Let $\Gamma \subset \partial \Omega$ be relatively open and let $T>0$. For $0<\varepsilon \leqslant T$, assume that for every $f$ with a singular support in $(0, \varepsilon) \times \Gamma, \Lambda f$ is known on $(0, T) \times \Gamma$, up to a smooth function. Then the lens relations $\left(L_{p}, \ell_{p}\right)$ and $\left(L_{s}, \ell_{s}\right)$ are determined uniquely on the open sets of $(x, v)$ with $x \in \Gamma$ so that the unit speed geodesic issued from $(x, v)$ (i.e. unit speed in the direction of $v$ ) in the metric $c_{p}^{-2} \mathrm{~d} x^{2}$, respectively $c_{s}^{-2} \mathrm{~d} x^{2}$, is transversal at $x$ and hits $\partial \Omega$ again, transversely, at a point in $\Gamma$ at a time not exceeding $T$.

Proof. By [18], the jets of $\rho, \lambda, \mu$ at $\Gamma$ are uniquely determined by the kernel of $\Lambda$ known on $[(0, \varepsilon) \times \Gamma]^{2}$ for any fixed $\varepsilon>0$. Since the proof is based on applying $\Lambda$ to highly oscillatory functions, any smooth addition to that kernel would not change the reconstruction.

Using this, we extend $\rho, \lambda, \mu$ smoothly to some small neighborhood $U$ of $\Gamma$ in the exterior $\Omega_{\text {ext }}$ of $\bar{\Omega}$ in a way determined uniquely by the data in the theorem.

Choose $\zeta_{1}:=\left(t_{1}, x_{1}, \tau_{1}=-c_{p}\left|\xi^{1}\right|, \xi^{1}\right) \in T^{*}((0, \varepsilon) \times \bar{\Omega}) \backslash 0$ (which is characteristic for the Hamiltonian related to $c_{p}$ ), with $x_{1} \in \partial \Omega$ and $\xi^{1}$ pointing into $\Omega$. Assume that the null bicharacteristic $\gamma$ in $\bar{\Omega}$ (actually, $T^{*}(\mathbf{R} \times \bar{\Omega})$ ) through $\zeta_{1}$ is transversal at that point, and hits $T^{*}(\mathbf{R} \times \partial \Omega)$ again transversely at some $\zeta_{2}:=\left(t_{2}, x_{2}, \tau_{2}, \xi^{2}\right)$ with $x_{2} \in \partial \Omega$. Extend $\gamma$ outside the domain on the side of $\zeta_{1}$ until it hits $\{t=0\}$ at some point $\zeta_{0}:=\left(t=0, x_{0}, \tau_{0}=-c_{p}\left|\xi^{0}\right|, \xi^{0}\right)$. If $0<\varepsilon \ll 1$, this short segment will be outside $\bar{\Omega}$, i.e. $x_{0} \notin \bar{\Omega}$, see figure 2 .

Let $u_{0}^{\prime}$ be an outgoing ( mooth for $t<0$ in $\Omega$ ) microlocal solution in $(0, T) \times \Omega_{\text {ext }}$ related to $(\rho, \lambda, \mu)$, with a wave front set in $\Sigma_{p}$ only, the latter supported in a small conic neighborhood of $\gamma$. This solution can be constructed by choosing suitable Cauchy data at $t=0$ near $x_{0}$ as explained in the previous section. We think of $u_{0}^{\prime}$ as a microlocal p-wave propagating along $\gamma$. We cut smoothly $u_{0}^{\prime}$ so that its support is concentrated near $\gamma$; call the result $u_{0}$. Then $\left(\partial_{t}^{2}-E\right) u_{0}=v \in C^{\infty}\left((0, T) \times \Omega_{\text {ext }}\right)$ and $u_{0}=\partial_{t} u_{0}=0, v=0$ in $\Omega$ for $t$ near 0 .

The trace $f$ of $u_{0}$ on the boundary can be naturally written as $f=f_{1}+f_{2}$, where $f_{j}$ have wave front sets in small conic neighborhoods of the projection of $\gamma$ on $T^{*}(\mathbf{R} \times \partial \Omega)$, i.e. close to $\zeta_{j}^{\prime}:=\left(t_{j}, x_{j}, \tau_{j},\left(\xi^{j}\right)^{\prime}\right), j=1,2$, where the prime stands for a tangential projection. Note that the role of $u_{0}$ was to generate $f_{1}$, giving rise to microlocal $p$-waves only.

Let $u$ solve (3) with $f=f_{1}$ (i.e. $f_{2}$ is replaced by zero). The singularities issued from $f_{1}$ will propagate to the future only and before they hit $\partial \Omega$ again, $u_{0}$ and $u$ differ by a smooth function. When they hit $\partial \Omega$, they will reflect at $\partial \Omega$ and there will be a possible mode conversion. We are not going to build a parametrix for the reflection. Instead, it is enough to prove that $\partial_{\nu} u$ has a non-empty wave from set in a conic neighborhood of $\zeta_{2}^{\prime}$.

Let $w$ be $u$ extended as zero to $\Omega_{\text {ext }}$. Then $\left(\partial_{t}^{2}-E\right) w=-\rho^{-1}(N u) \otimes \delta_{\mathrm{b}}$, where $\delta_{\mathrm{b}}$ is the delta function on $\mathbf{R} \times \partial \Omega$ on the boundary, see (4). Assume $\zeta_{2}^{\prime} \notin \mathrm{WF}(N u)$; then at $\left(t_{2}, x_{2}\right)$, the 
wave front set of $-(N u) \otimes \delta_{\mathrm{b}}$ on the plane $\pi$ spanned by $\zeta_{2}^{\prime}$ and the conormal to the boundary can be only along the conormal, as it follows by the calculus of the wave front sets. In particular, $\left(\tau_{2}, \xi^{2}\right)$ (the fiber component of $\gamma$ at that point) cannot be in the wave front set of $(N u) \otimes \delta_{\mathrm{b}}$ because the latter is in $\pi$; and $\left(\tau_{2}, \xi^{2}\right)$ is certainly not conormal, being characteristic. By the propagation of singularities theorem (with a source term having a wave front set away from the microlocal region of interest), each point of $\gamma$ must be a singularity for $w$, or none is. This is a contradiction since $w$ is singular on $\gamma$ in the domain, and non-singular on $\gamma$ outside it. Therefore, $\zeta_{2}^{\prime} \in \mathrm{WF}(\mathrm{Nu})$.

We can take a sequence of $f$ 's as above with shrinking wave fronts sets to $\zeta_{1}$ (or take $f$ with $\mathrm{WF}\left(f_{1}\right)$ on the radial ray through $\left.\zeta_{1}\right)$ to conclude that $\Lambda$ determines the p-lens relation $\left(L_{p}, \ell_{p}\right)$ at $\zeta_{0}$. Since the part of $\gamma$ between $\zeta_{0}$ and $\zeta_{1}$ is uniquely determined by the data, conclude that $\left(L_{p}, \ell_{p}\right)$ is uniquely determined at $\zeta_{1}$ as well.

To show that $\Lambda$ determines the lens relation $\left(L_{s}, \ell_{s}\right)$ related to the s-waves on $\mathcal{G}_{s}$, we argue as above.

Remark 5.1. The proof actually shows that we only need to know the wave front set of the kernel of $\Lambda$ microlocally at $\left(\left(t_{1}, x_{1}, \tau_{1},-\left(\xi^{1}\right)^{\prime}\right),\left(t_{2}, x_{2}, \tau_{2},\left(\xi^{2}\right)^{\prime}\right)\right.$ only for $c_{p}$ and similarly for $c_{s}$, in order to decide if $\left(x_{1},\left(\xi^{1}\right)^{\prime}, x_{2},\left(\xi^{2}\right)^{\prime}, t_{2}-t_{1}\right)$ belongs to the graph of the p-lens relation with an identification of covectors and vectors by the metric $c_{p}^{-2} \mathrm{~d} x^{2}$. Here, $\left(t_{2}, x_{2}, \tau_{2},\left(\xi^{2}\right)^{\prime}\right)$ is the image of $\left(t_{1}, x_{1}, \tau_{1}, \xi^{1}\right)$ (recall that $\left(\xi^{1}\right)^{\prime}$ is the projection of $\left.\xi^{1}\right)$ under the bicharacteristic flow until it hits the boundary; and then projected there.

Proof of theorem 1. Consider $c_{p}$ first. By theorem 2, we can recover the lens relation $\left(L_{p}, \ell_{p}\right)$ on the foliation. By [23], this recover $c_{p}$ in the region covered by the foliation, as claimed. The proof for $c_{s}$ is the same.

\section{The Herglotz and Wieckert and Zoeppritz condition}

We formulate generalized version of the Herglotz [12] and Wieckert and Zoeppritz [30] condition on a speed $c(x)$ in the ball $B(0, R)$ :

$$
\frac{\partial}{\partial r} \frac{r}{c(r, \omega)}>0, \quad \text { for } 0<r=|x| \leqslant R,
$$

where $x=r \omega,|\omega|=1$ and we work in $\mathbf{R}^{n}$ in this section. The original condition in $[12,30]$ is about radial speeds $c(r)$ only. In particular, (23) holds when $\partial_{r} c<0$, i.e. when the speed decreases in depth. This inequality was shown on [23] to be equivalent to the requirement the Euclidean sphere $|x|=r$ to be strictly convex with respect to $c^{-2} \mathrm{~d} x^{2}$. If $\partial \Omega$ is flat locally, the convexity condition is that $c$ increases with depth. We formulate those two conditions formally in the following.

\section{Lemma 2.}

(a) The Euclidean spheres $S_{r}=\left\{x \in \mathbf{R}^{n} ;|x|=r\right\}, R_{1} \leqslant r \leqslant R_{2}$, form a strictly convex foliation in some set with respect to the metric $c^{-2} \mathrm{~d} x^{2}$, viewed from the exterior, if and only if (23) holds for such $r$ and for $x=r \omega$ in that set.

(b) The Euclidean hyperplanes $\left\{x \in \mathbf{R}^{n} ; x^{n}=C\right\}, C_{1} \leqslant C \leqslant C_{2}$ form a strictly convex foliation in some set with respect to the metric $c^{-2} \mathrm{~d} x^{2}$, viewed from $x^{n}>C_{2}$ if and only $\partial c / \partial x^{n}>0$ in that set. 

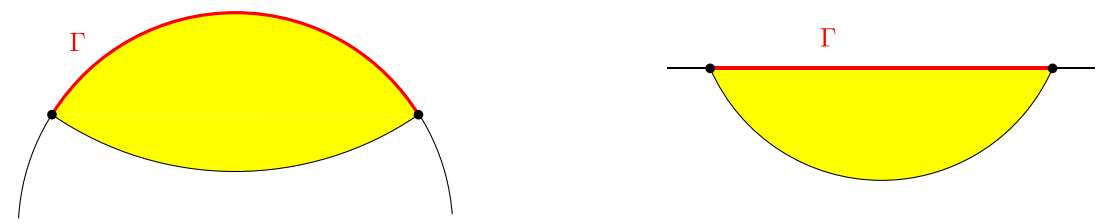

Figure 3. The shaded region is where we can recover the speed if the speed increases with depth, illustrating lemma 2 (a) and (b), respectively.

Part (a) is proved in [23]. Those two statements are a partial case of the following more general one. Recall that strict convexity of an oriented hypersurface $S$ in a Riemannian manifold is defined as a positivity of the second fundamental form; and if that form in non-negative, we will call $S$ convex. If the second fundamental form vanishes at some point of $S$, we call this point flat, which is a special case of convex. Under this definition, totally geodesic hypersurfaces are still convex.

Lemma 3. Let the oriented hypersurface $S$ be strictly convex w.r.t. the metric $g$ at some point $x_{0}$. Fix a smooth $c>0$. Let $\partial / \partial \nu$ be the unit normal derivative at $x_{0}$ pointing to the convex side. If $\partial c / \partial \nu<0$, then $S$ is strictly convex w.r.t. the metric $c^{-2} g$ at $x_{0}$.

If $S$ is flat at $x_{0}$, then $\partial c / \partial \nu<0$ is an if and only if condition for strict convexity.

Proof. We work in semigeodesic local coordinates near $x_{0}$ so that $S$ is given locally by $x^{n}=0$ and the convex side is $x^{n}>0$. We need to show that the second fundamental form related to $c^{-2} g$ on hyperplane $x^{n}=0$ is positive when that related to $g$ is. Denote the Christoffel symbols of $g$ by $\Gamma_{i j}^{k}$ and those of $c^{-2} \mathrm{~d} x^{2}$ by $\tilde{\Gamma}_{i j}^{k}$. Using the relationship between Christoffel symbols of conformal metrics, we get

$$
\tilde{\Gamma}_{i j}^{k}=\Gamma_{i j}^{k}+\frac{1}{2} c^{2}\left(\delta_{j}^{k} \partial_{x^{i}}+\delta_{i}^{k} \partial_{x^{j}}-g_{i j} \partial_{x^{k}}\right) c^{-2} .
$$

On $T\left\{x^{n}=0\right\}$, which implies $\xi^{n}=0$ in particular, the second fundamental form of $g$ and $c^{-2} g$ on $x^{n}=0$ are related by

$$
-\tilde{\Gamma}_{\alpha \beta}^{n} \xi^{\alpha} \xi^{\beta}=-\Gamma_{\alpha \beta}^{n} \xi^{\alpha} \xi^{\beta}+\frac{1}{2} c^{2}\left|\xi^{\prime}\right|_{g}^{2} \partial_{x^{n}} c^{-2},
$$

where Greek indices run from 1 to $n-1$ and $\xi^{\prime}=\left(\xi^{1}, \ldots, \xi^{n-1}\right)$. Therefore, that form is positive if $\partial_{x^{n}} c<0$; and when the form on the right vanishes, then this is an if and only of condition.

Lemma 2(b) then follows from lemma 3 since the Euclidean hypersurfaces are convex by our definition (they are flat). Using the fact that the Euclidean spheres are strictly convex, we can derive strict convexity in lemma 2(a) under the weaker condition $\partial_{r} c<0$. One can check directly the the Euclidean spheres are flat for the metric $|x|^{-2} \mathrm{~d} x^{2}$, which implies lemma 2(a) in its full strength.

Part (b) and theorem 1 in particular provide uniqueness for the local seismology problem when the surface of the Earth is modeled as the plane $x^{3}=0$ in $\mathbf{R}^{3}$, and the Earth itself is given locally by $x^{3}>0$, under the conditions $\partial_{x^{3}} c_{p}>0$ and $\partial_{x^{3}} c_{s}>0$, see figure 3(b). For deeper regions, the spherical model can be used and then condition (23) guarantees existence of a strictly convex foliation. Those conditions are satisfied in the upper mantle, at least, according 
to the popular preliminary reference Earth model (PERM) [9]. In fact, the stronger condition $\partial_{r} c<0$ holds, and figure 3(a) illustrates typical regions where the uniqueness holds.

\section{ORCID iDs}

Plamen Stefanov (i) https://orcid.org/0000-0002-8544-3411

\section{References}

[1] Bao G and Zhang H 2014 Sensitivity analysis of an inverse problem for the wave equation with caustics J. Am. Math. Soc. 27 953-81

[2] Belishev M I 1987 An approach to multidimensional inverse problems for the wave equation Dokl. Akad. Nauk SSSR 297 524-7

[3] Belishev M I 2006 Dynamical inverse problem for a Lamé type system J. Inverse Ill-Posed Problems 14 751-66

[4] Belishev M I 2007 Recent progress in the boundary control method Inverse Problems 23 R1-67

[5] Belishev M I and Kurylev Y V 1992 To the reconstruction of a Riemannian manifold via its spectral data (BC-method) Commun. PDE 17 767-804 MR1177292

[6] Bellassoued M and Dos Santos Ferreira D 2011 Stability estimates for the anisotropic wave equation from the Dirichlet-to-Neumann map Inverse Problem Imaging 5 745-73

[7] Bosi R, Kurylev Y and Lassas M 2016 Stability of the unique continuation for the wave operator via Tataru inequality and applications J. Differ. Equ. 260 6451-92

[8] Dencker N 1982 On the propagation of polarization sets for systems of real principal type J. Funct. Anal. 46 351-72

[9] Dziewonski A M and Anderson D L 1981 Preliminary reference earth model Phys. Earth Planet. Inter. 25 297-356

[10] Eller M, Isakov V, Nakamura G and Tataru D 2002 Uniqueness and stability in the Cauchy problem for Maxwell and elasticity systems Nonlinear Partial Differential Equations and Their Applications. Collège de France Seminar, vol XIV (Paris, 1997/1998) (Studies in Mathematics and its Applications vol 31) (Amsterdam: North-Holland) pp 329-49

[11] Hansen S and Uhlmann G 2003 Propagation of polarization in elastodynamics with residual stress and travel times Math. Ann. 326 563-87

[12] Herglotz G 1905 Über die Elastizitaet der Erde bei Beruecksichtigung ihrer variablen Dichte $Z$. Math. Phys. 52 275-99

[13] Katchalov A, Kurylev Y and Lassas M 2001 Inverse Boundary Spectral Problems (Chapman and Hall/CRC Monographs and Surveys in Pure and Applied Mathematics vol 123) (Boca Raton, FL: Chapman and Hall)

[14] Laurent C and Léautaud M Quantitative unique continuation for operators with partially analytic coefficients. Application to approximate control for waves. to appear in J. Eur. Math. Soc.

[15] Lechleiter A and Schlasche J W 2017 Identifying Lamé parameters from time-dependent elastic wave measurements Inverse Problems Sci. Eng. 25 2-26

[16] Monard F 2014 Numerical implementation of geodesic x-ray transforms and their inversion SIAM J. Imaging Sci. 7 1335-57

[17] Montalto C 2014 Stable determination of a simple metric, a covector field and a potential from the hyperbolic Dirichlet-to-Neumann map Commun. PDE 39 120-45

[18] Rachele L V 2000 Boundary determination for an inverse problem in elastodynamics Commun. PDE 25 1951-96

[19] Rachele L V 2000 An inverse problem in elastodynamics: uniqueness of the wave speeds in the interior J. Differ. Equ. 162 300-25

[20] Rachele L V 2003 Uniqueness of the density in an inverse problem for isotropic elastodynamics Trans. Am. Math. Soc. 355 4781-806 (electronic)

[21] Stefanov P 2000 Lower bounds of the number of the Rayleigh resonances for arbitrary body Indiana Univ. Math. J. 49 405-26 
[22] Stefanov P and Uhlmann G 2005 Stable determination of generic simple metrics from the hyperbolic Dirichlet-to-Neumann map Int. Math. Res. Not. 17 1047-61

[23] Stefanov P, Uhlmann G and Vasy A 2016 Boundary rigidity with partial data J. Am. Math. Soc. 29 299-332

[24] Stefanov P, Uhlmann G and Vasy A 2017 Local and global boundary rigidity and the geodesic x-ray transform in the normal gauge (arXiv 1702.03638)

[25] Stefanov P, Uhlmann G and Vasy A 2016 On the stable recovery of a metric from the hyperbolic DN map with incomplete data Inverse Problems and Imaging 10 1141-7

[26] Tataru D 1995 Unique continuation for solutions to PDE's D and Holmgren's theorem Commun. PDE 20 855-84

[27] Taylor M E 1981 Pseudodifferential Operators (Princeton Mathematical Series vol 34) (Princeton, NJ: Princeton University Press)

[28] Trèves F 1980 Introduction to pseudodifferential and Fourier integral operators Vol. 1 (Pseudodifferential operators, The University Series in Mathematics) (New York: Plenum)

[29] Uhlmann G and Vasy A 2016 The inverse problem for the local geodesic ray transform Inventiones Math. 205 83-120

[30] Wiechert E and Zoeppritz K 1907 Über Erdbebenwellen Nachr. Koenigl. Geselschaft Wiss. Göttingen 4 415-549 